Un compte rendu du travail figurera dans le Bulletin aussitôt après l'attribution du prix.

VIII. La Commission chargée de l'attribution du prix sera nommée par le Comité de Direction dans une séance tenue au mois de décembre qui suit la date du dépôt des travaux. Elle comprendra 9 membres ainsi répartis : 5 Membres du Comité de Direction et 4 nommés par la Société sur une liste présentée par le Comité et qui comprendra 8 noms.

$$
* *
$$

Le prix anonyme de 5.000 francs sera attribué pour la première fois en 1939 et au concours.

Le Comité de Direction a choisi le sujet suivant :

«Les processus de sensibilisation et d'immunisation dans les éptdermomycoses.

Les mémoires devront être adressés au Secrétaire général, Docteur Pierre Fernet, 11, rue de Sontay, Paris $\left(16^{\circ}\right)$ avant le 30 novembre 1938.

Le prix sera décerné à l'Assemblée générale de mai 1939.

\title{
Ouvrage reçu
}

Charles Franklin Craig et Ernest Carroll Faust. - Clinical Patasitology. Octavo, 733 pages, 243 figures. Relié, \$ 8,50 net. 1937. Lea \& Fibiger, Washington Square, Philadelphie, Pa., U.S.A.

\section{ERRATA}

Page $61,20^{\circ}$ ligne, au lieu de : Hauts de Breat, lire : Héaux de Bréhat. Page 70,12 ligne, au lieu de : le $P$., lire : de $P$.

Page 70, 18 ligne, avant Squalus, mettre 20.

Page 271, $19^{\text {e }}$ ligne, au lieu de : Chilossyllium, lire : Chiloscyllium.

Page 273, au lieu de : Chandhuri, lire : Chaudhuri.

Page $411,4^{\circ}$, $5^{\circ}$ et $6^{\circ}$ lignes, lire long au lieu de large.

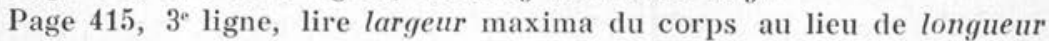
maxima du corps. 